\title{
ASPECTOS DA BIOLOGIA REPRODUTIVA DE Jatropha curcas L.
}

\author{
Reproductive biology aspects of Jatropha curcas $\mathbf{L}$.
}

\author{
Vespasiano Borges de Paiva Neto ${ }^{1}$, Jeruska Azevedo Moreira Brenha², Felipe Barbosa de Freitas ${ }^{2}$, \\ Mônica Cristina Rezende Zuffo², Rita de Cássia Félix Alvarez²
}

\begin{abstract}
RESUMO
O conhecimento da estrutura floral e da biologia reprodutiva numa cultura é básico para que o melhorista desenvolva técnicas de castração e polinização adequadas. Objetivando obter dados de biologia reprodutiva de pinhão manso, inflorescências emasculadas, foram observadas sem isolamento (controle positivo) e isoladas com tecido "nylon" tipo voal para posterior realização dos tratamentos de geitonogamia e xenogamia após a antese. Os tratamentos consistiram de controle positivo (flores não isoladas), e a partir das flores isoladas foram obtidos os demais tratamentos: controle negativo (sem polinização); xenogamia (receberam pólen de outra planta); geitonogamia 1 (receberam pólen da mesma inflorescência); geitonogamia 2 (receberam pólen de outra inflorescência da mesma planta). Os resultados indicam que a espécie não apresenta problemas de autoincompatibilidade, sendo os índices de fecundação bastante elevados e indiferentes estatisticamente em todos os tratamentos, com valores acima de $80 \%$, exceto para o controle negativo em que as inflorescências foram isoladas e não polinizadas, resultando em ausência de fecundação e de frutos. Esse último resultado mostra a importância da visitação de insetos para a obtenção de sucesso no processo reprodutivo da espécie. As flores masculinas, doadoras de pólen, abrem no período da manhã, assim como as femininas. Sendo assim, embora haja visitação de insetos durante todo o dia, o processo de polinização ocorre no período matutino, em razão da quase total ausência de pólen no período vespertino.
\end{abstract}

Termos para indexação: Jatropha curcas L., autofecundação, auto-incompatibilidade, polinização.

\begin{abstract}
The knowledge of the floral structure and reproductive biology are basic to a more successful for the development of techniques of castration and pollination in plant species. Aiming to obtain data about reproductive biology of physyc nut, free and enclosed inflorescences were observed without isolation (positive control) and isolation with nylon for posterior treatment of geitonogamy and xenogamy. So, treatments were positive control (free or unbagged inflorescences), and all other treatments obtained from isolated immature inflorescences enclosed within of fine nylon bags: negative control (without pollination), xenogamy (receiving pollen from another plant); geitonogamy 1 (received pollen from the same inflorescence), geitonogamy 2 (received pollen from another inflorescence of the same plant). The results indicate that the species does not present problems of self-incompatibility resulting in high fertilization and statistically indifferent in all treatments, presenting values above $80 \%$, except for negative control in which the inflorescences were isolated and not pollinated resulting in lack of fertilization and fruit development. These results show the importance insects for success in the reproductive process of this species. The male flowers, pollen donors, opened in the morning, just like the female ones. Thus, although there is visitation of insects during all the day, the process of pollination occurs in the morning, and almost total absence of pollen was observed after noon.
\end{abstract}

Index terms: Jatropha curcas L., self-pollination, self-incompatibility, pollination.

(Recebido em 3 de julho de 2009 e aprovado em 25 de janeiro de 2010)

\section{INTRODUÇÃO}

Espécies vegetais que podem ser processadas para fornecer um substituto para o óleo diesel derivado do petróleo têm atraído maior interesse dos cientistas de zonas temperadas do que aqueles de zonas tropicais (Heller, 1996). Nessa categoria de plantas, as seguintes propriedades tropicais do pinhão manso (Jatropha curcas L.) Euphorbiaceae, atraíram grande interesse de várias agências de desenvolvimento: boa adaptação a ambientes semi-áridos, seu óleo pode ser processado para o uso como um substituto do diesel combustível e podem ser usados para o controle da erosão. Por isso, um grande número de informações referentes à espécie precisam ser geradas, envolvendo caracterizações fenológica, reprodutiva e genotípica, técnicas de propagação, criação e manutenção de banco de germoplasma, metodologias para extração e composição do óleo, dentre outras. De acordo com Achten et al. (2008), o interesse no uso de Jatropha curcas L. como matéria-prima para a produção de biodiesel está crescendo rapidamente. Com isso, a espécie tem sido alvo de um número cada vez maior de trabalhos científicos nas mais variadas áreas do conhecimento em âmbito

${ }^{1}$ Universidade Federal de Mato Grosso do Sul/UFMS - Cx. P. 112 - 79560-000 - Chapadão do Sul, MS - vespasiano@nin.ufms.br

${ }^{2}$ Universidade Federal de Mato Grosso do Sul/UFMS - Campus de Chapadão do Sul - Chapadão do Sul, MS 
Internacional (Devanesan et al., 2007; Rao et al., 2007; Debnath \& Verma, 2008; Kochhar et al., 2008; Nunes et al., 2008; Peace \& Aladesanmi, 2008). Saturnino et al. (2005) e Achten et al. (2008) publicaram artigos revisando aspectos gerais da cultura.

Em plantas superiores, a transição da fase vegetativa para a fase reprodutiva envolve fatores exógenos (tais como fotoperíodo e temperatura) e endógenos (nível endógeno de fitohormônios e controle genético). $\mathrm{O}$ conhecimento da estrutura floral e da biologia reprodutiva numa cultura é básico para que o melhorista desenvolva técnicas de castração e polinização adequadas (Gomes et al., 2001; Silva et al., 2001). Segundo Damião Filho (1993), o rápido progresso alcançado no melhoramento das plantas cultivadas se deve aos estudos dos processos reprodutivos que ocorrem nas flores.

Alguns fatores característicos da espécie merecem especial atenção para futuros programas de melhoramento genético, como a necessidade de aumento do número de flores femininas por inflorescência, teor de óleo na semente, maior sincronismo na diferenciação floral e, consequente antese por inflorescência. Senthyl et al. (2008), Sunil et al. (2008) e Popluechai et al. (2009) publicaram trabalhos recentemente visando à caracterização ou melhoramento genético de pinhão manso.

Dados da literatura mostram que a abertura das flores masculinas e femininas pode ocorrer em períodos diferentes. Durante experimentações de campo, Heller (1996) observou um número de insetos diferentes que visitaram flores e realizaram polinização. Em Senegal, observou que as flores estaminadas abrem mais tarde do que flores pistiladas da mesma inflorescência. Com esta estratégia, a espécie desenvolveu um mecanismo que privilegia a polinização cruzada. Münch \& Kiefer (1989) não observou este comportamento cronológico em Cabo Verde, sugerindo que o mecanismo é influenciado pelo ambiente.

Neste trabalho, objetivou-se gerar informações sobre a biologia reprodutiva, essenciais para futuros programas de melhoramento genético de pinhão manso.

\section{MATERIAL E MÉTODOS}

As plantas utilizadas na realização do trabalho fazem parte do banco de germoplasma estabelecido há dois anos, em área cedida pela Fundação de Apoio à Pesquisa Agropecuária de Chapadão localizada no município de Chapadão do Sul-MS (Latitude:1841'33"sul Longitude:52 40’45"oeste; Altitude: $810 \mathrm{~m}$; precipitação média anual: $1850 \mathrm{~mm}$; Temperaturas médias anuais: mínima de 13 e máxima de $28^{\circ} \mathrm{C}$ ). Os experimentos foram conduzidos no período de outubro de 2008 a abril de 2009.
Para análise do sistema reprodutivo, foi realizada a emasculação, com a eliminação das flores masculinas, seguida do isolamento de inflorescências imaturas com tecido "nylon" tipo voal (porosidade de $0,1 \mathrm{~mm}^{2}$ ) para verificar a ocorrência de geitonogamia e xenogamia na espécie. A verificação da geitonogamia ocorreu em dois tratamentos distintos: geitonogamia 1 (polinização realizada com pólen proveniente de flores masculinas da mesma inflorescência) e geitonogamia 2 (polinização realizada com pólen proveniente de flores masculinas de outra inflorescência da mesma planta). A xenogamia foi verificada com a polinização das flores isoladas com pólen de outra planta. As flores foram polinizadas manualmente coletandose as flores masculinas e colocando as anteras em contato com o estigma das flores receptoras. Logo em seguida, as inflorescências foram novamente isoladas e etiquetadas, sendo os sacos retirados apenas após o desenvolvimento dos frutos. Para se observar a importância de insetos na reprodução da espécie, foram realizados dois controles: o controle negativo (flores isoladas e não polinizadas) e controle positivo (flores não isoladas).

$\mathrm{O}$ delineamento experimental foi inteiramente casualizado, com 5 repetições por tratamento, sendo cada repetição com cinco inflorescências, as quais possuíam em média 3,5 flores femininas. Os dados foram analisados estatisticamente (Tukey, 5\%) utilizando o programa estatístico Sisvar (Ferreira, 1999). Foram utilizadas 125 inflorescências com 562 flores femininas no experimento, com média de 4,5 flores femininas por inflorescência.

A observação da morfologia floral quanto à presença de estruturas secretoras foi realizada após a coleta das flores em fase pré-antese, acondicionamento em frascos de vidro contendo água e transporte até o laboratório de anatomia vegetal do campus de Chapadão do Sul da Universidade Federal de Mato Grosso do Sul. Foram realizados cortes longitudinais à mão livre com auxílio de lâmina de barbear em flores masculinas e femininas. As imagens foram obtidas com auxílio de câmera fotográfica digital (Sony ${ }^{\circledR}$ - DSC H10).

\section{RESULTADOS E DISCUSSÃO}

O processo de polinização natural em pinhão manso é realizado por pequenos insetos (Figura 1C) e que embora as flores recebam visitação ao longo de todo o dia, o período matutino constitui-se no mais importante para polinização, uma vez que a antese ocorre ao amanhecer e que a disponibilidade de pólen reduz significativamente ao longo da manhã. Essa observação está de acordo com Solomon Raju \& Ezradanam (2002), que relatam sobre abertura de flores de pinhão entre 5:50 e 6:30 da manhã em experimento realizado na Índia. 
Os resultados indicam que a espécie não apresenta problemas de auto-incompatibilidade, resultando em elevados índices de fecundação nos processos de geitonogamia, independente se a flor doadora de pólen pertence à mesma inflorescência ou a outra inflorescência da mesma planta (Tabela 1). Elevados índices de fecundação foram apresentados também com a realização de xenogamia (polinização cruzada). Solomon Raju \& Ezradanam (2002) obtiveram 96 e $77 \%$ de fecundação e desenvolvimento de frutos de Jatropha curcas ao realizarem processos de xenogamia e geitonomia, respectivamente. Bhattacharya et al. (2005) relatam valores inferiores de taxa de fecundidade natural $(50 \%)$ em comparação àquela encontrada no presente trabalho. A importância dos insetos no processo de polinização natural fica evidente quando comparamos os dados dos tratamentos em que a flor foi isolada (Figura 1B) para evitar a visitação de insetos (fecundidade nula) com aquela apresentada pelas inflorescências do controle positivo, em que houve livre visitação dos insetos (Figura 1C), resultando em taxa média de fecundação de $80,7 \%$ (Tabela 1). Heller (1996) e Saturnino et al. (2005) afirmam tratar-se de uma planta de fecundação cruzada e entomófila. Solomon Raju \& Ezradanam (2002) listam formigas, abelhas, moscas, tripes e outros insetos como principais polinizadores, sendo a importância de cada tipo de inseto dependente do local em observação. Dehgan (1984) relata a presença, ainda que rara, de flores hermafroditas em pinhão manso. No entanto, em nossas observações nenhuma flor hermafrodita foi detectada nas plantas da área experimental.

A abertura das flores masculinas inicia-se antes das flores femininas. No entanto, como o número de flores masculinas é bem maior do que o número de flores femininas (Figura 1A), durante todo o período de antese das flores femininas haviam flores masculinas abertas. Dessa forma, a geitonogamia pode ocorrer naturalmente.
Dados da literatura mostram que a abertura das flores masculinas e femininas pode ocorrer em períodos diferentes. Na Índia, relatos de Solomon Raju \& Ezradanam (2002), mostram que as flores masculinas permanecem abertas por 11 dias e que as flores femininas abrem do segundo ao sexto dia em relação às masculinas. Já, na região de Januária - MG, Saturnino et al. (2005) afirmam que as flores femininas abrem antes das masculinas dentro de uma mesma inflorescência, demonstrando total falta de sincronia

O processo de emasculação das inflorescências é bastante simples nos dias que precedem a antese das flores, dadas as diferenças morfológicas das flores pistiladas e estaminadas (Figuras 1A, 1D, 1E, 1F), sendo as flores femininas marcadamente maiores e apresentando, em relação às flores masculinas, aproximadamente o dobro do diâmetro do pedúnculo $(0,2$ e $0,1 \mathrm{~cm}$, respectivamente). Dehgan (1984) observou que a rotina padrão de emasculação e ensacamento de inflorescências de pinhão manso deve ser necessária para evitar a autopolinização, em casos em que as flores estaminadas e pistiladas abram simultaneamente, a emasculação pode ser requerida.

Foi possível observar a presença de glândulas livres na base do ovário em flores femininas (Figura 1D) e na base dos estames em flores masculinas (Figuras 1D e 1E). Saturnino et al. (2005) também relatam a presença desta estrutura em Jatropha curcas L. Por outro lado, Santos et al. (2005) observaram várias diferenças entre as flores masculinas e femininas de Jatropha mutabilis (Pohl) Baill. e Jatropha mollissima (Pohl) Baill. O nectário das flores femininas das espécies estudadas é um disco, localizado na base do ovário, onde o néctar se acumula. O nectário das flores masculinas de J. mutabilis é formado por cinco glândulas soldadas na base, envolvendo a porção basal dos filetes. Nas flores masculinas de J. mollissima o nectário também é constituído por cinco glândulas que, no entanto, encontram-se livres.

Tabela 1 - Frutificação em flores de Jatropha curcas L. polinizadas artificialmente. Chapadão do Sul-MS, 2009.

\begin{tabular}{lc}
\hline Tratamento & Frutificação (\%) \\
\hline Controle negativo & $00,0 \mathrm{~b}$ \\
Controle positivo & $80,7 \pm 6,7 \mathrm{a}$ \\
Geitonogamia 1 (pólen mesma inflorescência) & $80,8 \pm 10,4 \mathrm{a}$ \\
Geitonogamia 2 (pólen inflorescências diferentes) & $90,5 \pm 7,6 \mathrm{a}$ \\
Xenogamia & $88,8 \pm 10,3 \mathrm{a}$ \\
\hline
\end{tabular}

Médias seguidas de mesma letra não diferem entre si pelo teste de Tukey (5\%). 

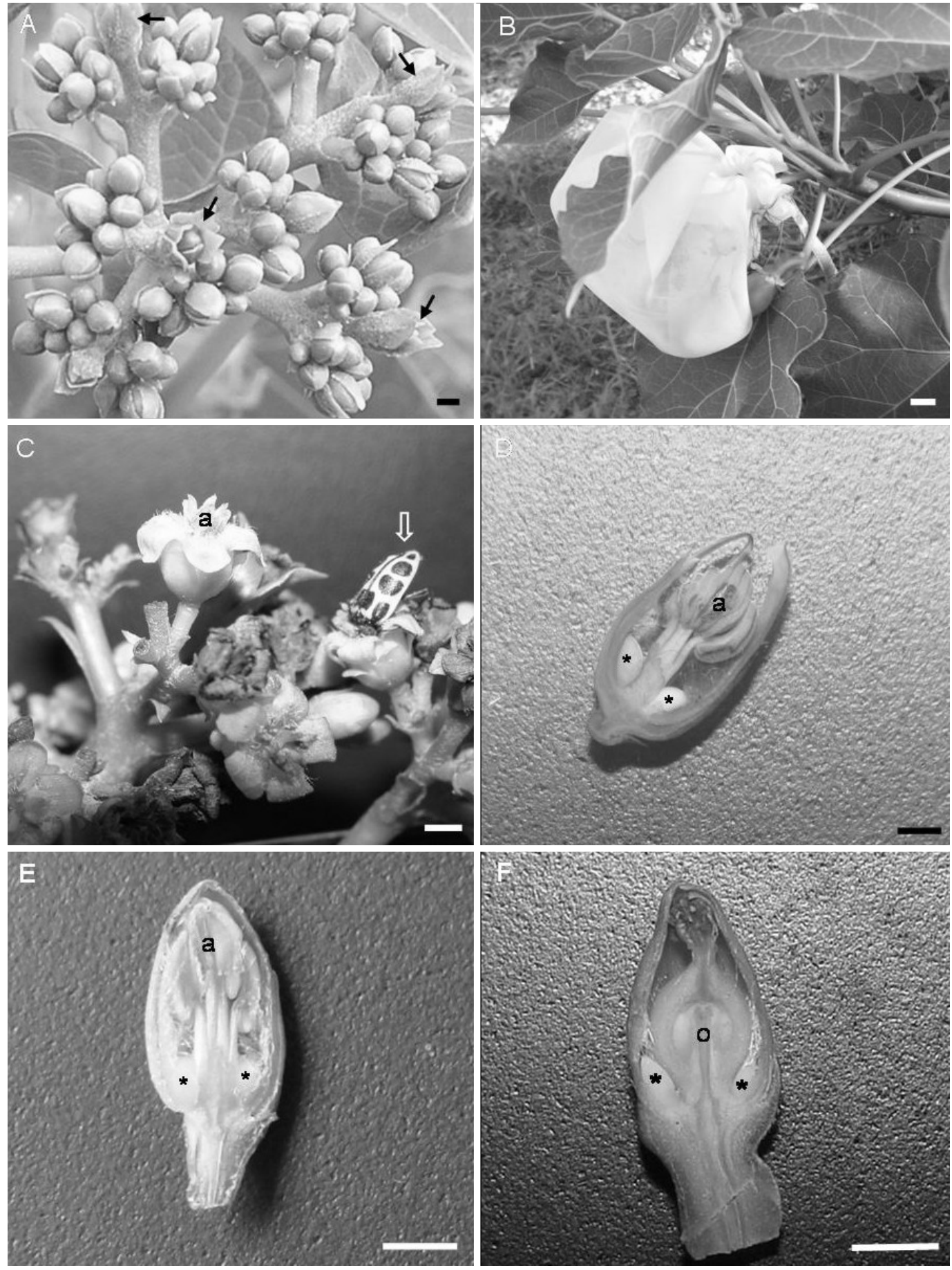

Figura 1 - Fotos ilustrativas do experimento e da morfologia floral de Jatropha curcas L. A - Inflorescência em fase de emasculação; B - Inflorescência isolada após emasculação; C - Larva angorá Astylus variegatus (Germar, 1824) (Coleoptera, Melyridae) visitando inflorescência masculina; D e E - Corte longitudinal de flor masculina; F - Corte longitudinal de flor feminina. Barras: $0,4 \mathrm{~cm}$ (B) e 0,2 cm (demais figuras); $\Rightarrow=$ flor feminina; $\mathrm{a}=$ anteras; $\mathrm{o}=\mathrm{ovário;} *$ glândulas florais. 


\section{CONCLUSÕES}

As diferenças morfológicas permitem a fácil emasculação em inflorescências de pinhão manso;

As primeiras horas da manhã constituem-se no melhor período para realização de polinização em pinhão manso;

Ocorre fecundação nos processos de geitonogamia e xenogamia em Jatropha curcas L., não havendo autoincompatibilidade na reprodução sexuada;

A presença de insetos é fundamental para a ocorrência de polinização em pinhão manso.

\section{AGRADECIMENTOS}

À Universidade Federal de Mato Grosso do Sul, pela bolsa de iniciação concedida ao primeiro autor e auxílio financeiro. À Fundação de Apoio à Pesquisa Agropecuária de Chapadão, pela manutenção da área experimental.

\section{REFERÊNCIAS BIBLIOGRÁFICAS}

ACHTEN, W.M.J.; VERCHOT, L.; FRANKEN, Y.J.; MATHIJS, E.; SINGH, V.P.; AERTS, R.; MUYS, B. Jatropha bio-diesel production and use. Biomas and Bioenergy, Oxford, v.32, n.12, p.1063-1084, 2008.

BHATTACHARAYA, A.; KALYANI, D.; SUBODH, K.D. Floral biology, floral resource constraints and Pollination Limitation in Jatropha curcas L. Pakistan Journal of Biological Sciences, Faisalabad, v.8, n.3, p.456-460, 2005.

DAMIÃO FILHO, C.F. Morfologia vegetal. Jaboticabal: Funep; Unesp, 1993. 243p.

DEBNATH, M.; VERMA, H.N. Effect of phytoprotein treatment on Jatropha curcas for wasteland reclamation effect of phytoprotein treatment on Jatropha curcas for wasteland reclamation. African Journal of Biotechnology, Nairobi, v.7, n.5, p.613-616, 2008.

DEHGAN, B. Phylogenetic significance of interspecific hybridization in Jatropha (Euphorbiaceae). Systematic Botany, Washington, v.9, n.4, p.467-478, 1984.

DEVANESAN, M.G.; VIRUTHAGIRI, T.; SUGUMAR, N. Transesterification of Jatropha oil using immobilized Pseudomonas fluorescens. African Journal of biotechnology, Nairobi, v.6, n.2, p.24972501, 2007.
FERREIRA, D.F. SisVar - Sistema de Análise de Variância para Dados Balanceados, versão 4.0. Lavras: UFLA, 1999. Software estatístico.

GOMES, J.E.; PAVANI, M.C.M.D.; PERECIN, D.; MARTINS, A.B.G. Morfologia floral e biologia reprodutiva de genótipos de aceroleira. Scientia Agrícola, Piracicaba, v.58, n.3, p.519-523, 2001.

HELLER, J. Physic nut. Jatropha curcas L. promoting the conservation and use of underutilized and neglected crops. Rome: Institute of Plant Genetics and Crop Plant Research, 1996. 66p.

KOCHHAR, S.; SINGH, S.P.; KOCHHAR, V.K. Effect of auxins and associated biochemical changes during clonal propagation of the biofuel plant - Jatropha curcas. Biomas and Bioenergy, Oxford, v.32, n.12, p.1136-1143, Dez. 2008.

MÜNCH, E.; KIEFER, J.F. Purging nut (Jatropha curcas L.) multiple use plant as a source of fuel in the future. Schriftenreihe der Gesellschaft fur Technishe Zusammenarbeit, Stuttgart, v.209, n.209, p.32, 1989.

NUNES, C.F.; PASQUAL, M.; SANTOS, D.N.; CUSTÓDIO, T.N.; ARAÚJO, A.G. Diferentes suplementos no cultivo in vitro de embriões de pinhãomanso. Pesquisa Agropecuária Brasileira, Brasília, v.43, n.1, p.9-14, jan. 2008.

PEACE, O.E.; ALADESANMI, A.O. Effect of fermentation on some chemical and nutritive properties of Berlandier Nettle Spurge (Jatropha cathartica) and physic nut (Jatropha curcas) seeds. Pakistan Journal of Nutrition, Faisalabad, v.7, n.2, p.292-296, 2008.

POPLUECHAI, S.; BREVIARIO, D.; MULPURI, S.; MAKKAR, H.P.S.; RAORANE, M.; REDDY, A.R.; PALCHETTI, E.; GATEHOUSE, A.M.R.; SYERS, J.K.; O'DONNELL, A.G.; KOHLI, A. Narrow genetic and apparent phenetic diversity in Jatropha curcas: initial success with generating low phorbol ester interspecific hybrids. Nature precedings, London, 2009. Disponível em: idocuments/278 $2 /$ version/1/files/npre20092782-1.pdf $>$. Acesso em: 26 jun. 2009. 
RAO, D.M.; HUSSAIN, S.M.D.J.; RANGADU, V.P.; SUBRAMANYAM, K.; KRISHNA, G.S.; SWAMY, A.V.N. Fermentatative production of itaconic acid by Aspergillus terreus using Jatropha seed cake. African Journal of Biotechnology, Nairobi, v.6, n.18, p.2140-2142, 2007.

SANTOS, M.J.; MACHADO, I.C.; LOPES, A.V. Biologia reprodutiva de duas espécies de Jatropha L.

(Euphorbiaceae) em Caatinga, Nordeste do Brasil.

Revista Brasileira de Botânica, São Paulo, v.28, n.2, p.361-373, abr./jun. 2005.

SATURNINO, H.M.; PACHECO, D.D.; KAKIDA, J.; TOMINAGA, N.; GONÇALVES, N.P. Cultura do pinhãomanso (Jatropha curca L.). Informe Agropecuário, Belo Horizonte, v.26, n.229, p.44-78, 2005.

SENTHIL KUMAR, R.; PARTHIBAN, K.T.; GOVINDA RAO, M. Molecular characterization of Jatropha genetic resources through inter-simple sequence repeat (ISSR) markers. Molecular Biology Reports, Nov. 2008. Disponível em: <http://Www.springerlink.com/content/ $\overline{1} 1 \overline{4} 7405671 \overline{1} 1 \mathrm{w} 2 \mathrm{7}>$. Acesso em: 26 jun. 2009.

SILVA, R.M.; BANDEL, G.; FARALDO, M.I.F.; MARTINS, P.S. Biologia reprodutiva de etnovariedades de mandioca. Scientia Agrícola, Piracicaba, v.58, n.1, p.101-107, 2001.

SOLOMON RAJU, A.J.; EZRADANAM, V. Pollination ecology and fruiting behaviour in a monoecious species, Jatropha curcas L. (Euphorbiaceae). Current Science, Bangalore, v.83, n.11, p.1395-1398, Dec. 2002.

SUNIL, N.; VARAPRASAD, K.S.; SIVARAJ, N.; SURESH KUMAR, T.; ABRAHAM, B.; PRASAD, R.B.N. Assessing Jatropha curcas L. germplasm in-situ: a case study. Biomass and Bioenergy, Oxford, v.32, n.3, p.198202, Mar. 2008. 